\title{
Research of license plate recognition based on HSV Space
}

\author{
Ying Qian ${ }^{1, \text { a }}$, Sheng Jun Gan ${ }^{2, b}$ \\ ${ }^{1}$ Chongqing University of Posts and Telecommunications, Chongqing 400065, China; \\ ${ }^{2}$ Chongqing University of Posts and Telecommunications, Chongqing 400065, China. \\ a qianying@cqupt.edu.cn, b392564834@qq.com
}

\begin{abstract}
Keywords: License Plate Recognition, Color feature, Sobel operator, Edge Detection.
\end{abstract}
\begin{abstract}
In order to solve the problem of license plate recognition in a complex environment, put forward a combined with the feature of license plate color, gray features, geometric shape, a general method of license plate recognition, first of all according to the color feature of license plate of China, this paper proposes a method based on HSV color space color recognition of license plate, solves the basic problem of license plate recognition accuracy is not high; Then with the improved Sobel operator edge detection method to obtain a rectangular area where the background plate; Finally could license plate area unified dimension, and verification, the last segment the license plate area. Adopt natural complex images taken to validate the new method in the environment of experiments show that compared with other methods, the new method can be adaptive within limits the type of license plate number, size, and direction, and the car position in the image and image background of fewer restrictions, applicability is a strong way.
\end{abstract}

\section{Introduction}

Along with the continuous development of society, also an increasing number of motor vehicles owned by individuals, in this case, rely solely on developing traffic facilities have been unable to solve the traffic congestion, traffic accidents and other issues. Therefore, vehicle management has already become an important part of the intelligent traffic management, and license plate recognition system (LRP) has become one of the important research topics in the field of intelligent transportation, electronic police system, bayonet system, intelligent transportation reported in the station, parking and highway toll management system has been widely used. Comprising three aspects: location and extraction of the license plate, license plate tilt correction and character segmentation, character recognition, the license plate location and character recognition rate and accuracy is the key, directly affects the success or failure of the recognition system. Because the image is from the complex natural environment were taken, which such as license plate specifications, style diversity, the defacement of license plates, light dark and complex background of factors for license plate recognition add a certain degree of difficulty, so the system of the key technology of design has strong robustness and broad applicability of the algorithm, can significantly improve the success of license plate recognition rate.

Now, the research of license plate recognition has been gradually mature, and many scholars have put forward their own methods, summed up as follows:1 1he license plate recognition method based on texture feature, because the license plate is provided with a horizontal texture so the method mainly using license plate recognition, such as the horizontal gradient difference method[1], wavelet texture[2]; (2) the license plate recognition method based on mathematical morphology[3,4],this method mainly uses mathematical morphology to identify the license plate; (3) the license plate recognition method based on edge[5,6]; (4) the license plate recognition method based on color, because the color of the type of car license is limited, and the characters and the cards have obvious color difference, the method main advantage of these features for license plate recognition, which are commonly used methods such as color edge algorithm, the color distance and similarity algorithm[5] and so on distance similarity algorithm [1,7]. Overview of these methods is not difficult to find that they will have more or less limitations, but also by environmental conditions, the background image 
is more restricted, within a certain limit is not well adaptive plate type, size, number and direction, weak applicability.

Accordingly, this paper puts forward the different from most other recognition method of vehicle license plate, the method based on the HSV color space is a unique advantage and the color characteristics of the license plate, and integration of the binary image mathematical morphology processing method, has carried on the organic combination of license plate color feature and geometric feature, is a very good effect recognition method. And restrictions on the size and tilt of the plate in the image, the light conditions, the vehicle's position or degree background in the image of the complex factors such as relatively small, and therefore the proposed recognition method better, wider range of applications.

\section{License Plate Location Algorithm}

Selection threshold of each component of HSV. Because in the three components of hue $(\mathrm{H})$ and saturation (S), brightness (V) of the color space of HSV, only $\mathrm{H}$ associated with color.So when the value of $\mathrm{H}$ doesn't change and $\mathrm{S}$ and $\mathrm{V}$ components have little change, They represent the color range will be fixed.H value, when the value of $\mathrm{V}$ is not changed, we change the brightness of $\mathrm{V}$ and the saturation of $\mathrm{S}$, the saturation and brightness of color will change. When the equation of $\mathrm{V}=1$ and $\mathrm{S}=1$ have established, the color has the highest purity.Reduce the value of $\mathrm{S}$, color tends to become white. When the value of $\mathrm{V}$ is close to zero, the color will become dark. Therefore, the saturation of $\mathrm{S}$ and the brightness of $\mathrm{V}$ will affect the final color yet.So in this paper, we set $\mathrm{T}$ as a threshold.if the equation of $\mathrm{V} \geq \mathrm{T}$ and $\mathrm{S} \geq \mathrm{T}$ have established, the color is expressed by the $\mathrm{H}$ is the color of the final. This article selects $\mathrm{n}$ pieces of blue plate as an experimental example. Within the blue scope of the license,we select $m$ sample points as $P_{1}, P_{2} \ldots P_{m}$ 。 There are the results of the experment that the 800 pieces from different license plate images under the same condition. We can conclude the threshold scope of the three component of four color as shown in table 1.

Table1 threshold range of three components of four colors

\begin{tabular}{|c|c|c|c|c|}
\hline $\begin{array}{c}\text { Color } \\
\text { components }\end{array}$ & Blue & Yellow & White & Black \\
\hline $\mathrm{H}$ & {$\left[\begin{array}{ll}0.55 & 0.70\end{array}\right]$} & {$\left[\begin{array}{lll}0.06 & 0.10\end{array}\right]$} & - & - \\
\hline $\mathrm{S}$ & {$\left[\begin{array}{ll}0.35 & 0.75\end{array}\right]$} & {$\left[\begin{array}{ll}0.3 & 0.7\end{array}\right]$} & - & - \\
\hline V & {$\left[\begin{array}{ll}0.3 & 1.0\end{array}\right]$} & {$\left[\begin{array}{ll}0.3 & 1.0\end{array}\right]$} & {$\left[\begin{array}{ll}0.7 & 1.0\end{array}\right]$} & {$\left[\begin{array}{ll}0 & 0.25\end{array}\right]$} \\
\hline
\end{tabular}

Color primary location. With plate color is characterized by an initial positioning plate and select blue license plates for the experimental cases, its initial positioning algorithm steps are:

Step1. Given the RGB image into HSV space, because the affected light conditions, so the need for image histogram equalization.

Step2. Calculation hue (H), saturation (S), brightness (V) threshold range, if $200 \leq \mathrm{H} \leq 280$ and $0.35 \leq \mathrm{V} \leq 1 、 0.35 \leq \mathrm{S} \leq 1$ labeled as white pixels, whereas marked as black pixels.

Step3.Step2 binary image obtained by further processing, picking out the license plate of a circumscribed rectangle, treatment methods are closing operations and take contour.

Step4. To meet the requirements of the circumscribed rectangle corresponding template matching and identify license plates. In general, an image requires multiple template matching, in order to ensure that the colors are positioned out of the plate. Similarly, you can use this algorithm to identify other colors of the license plate, just to change the threshold range. From the experimental results, the color effect is relatively better positioned. However, only using the license plate color positioning accuracy is not enough, positioned at such light intensity is weak, or the body of the blue, there will be failures.

The improved Sobel operator secondary positioning. The method of edge detection[8] is the change of pixel image inspection in a certain area of gray, marking significant changes in digital 
image brightness. Image edge detection can greatly reduce the amount of data, and remove irrelevant information, save the important attributes of the image structure. The traditional Sobel edge detection operator only horizontal and vertical two direction template, you can indicate that the horizontal direction of the template in the horizontal direction of edge response of the largest maximum vertical template in vertical direction of edge response. In view of this, we improved the Sobel edge detection operator, put forward the following template:

$$
\left|\begin{array}{ccc}
-1 & -\sqrt{2} & 0 \\
-\sqrt{2} & 0 & \sqrt{2} \\
0 & \sqrt{2} & 1
\end{array}\right| \quad\left|\begin{array}{ccc}
0 & -\sqrt{2} & -1 \\
\sqrt{2} & 0 & -\sqrt{2} \\
1 & \sqrt{2} & 0
\end{array}\right| \quad\left|\begin{array}{ccc}
-1 & -\sqrt{2} & -1 \\
0 & 0 & 0 \\
1 & \sqrt{2} & 1
\end{array}\right| \quad\left|\begin{array}{rrr}
-1 & 0 & 1 \\
-\sqrt{2} & 0 & \sqrt{2} \\
-1 & 0 & 1
\end{array}\right|
$$

Point by point for the image is calculated, and take the maximum as a new image grey value, the improvement of the advantage of operator is interested in horizontal, vertical and diagonal direction of the image, more conducive to extract the image of the characters.

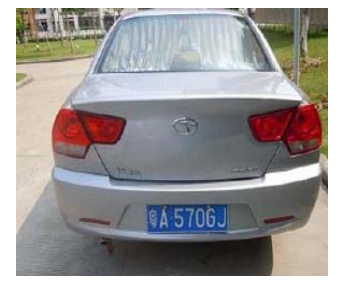

The license plate image

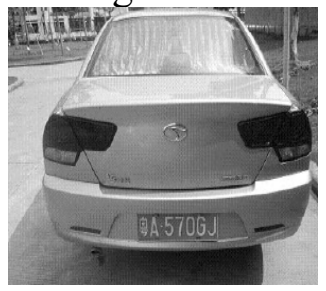

Gray change image

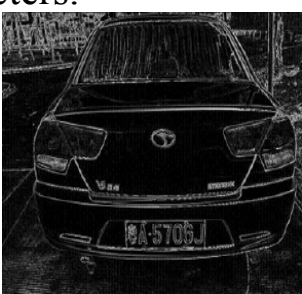

Sobel edge detection

Fig. 1 Sobel edge detection of rendering

Effect can be seen from the above experiment, in this paper, the improved edge detection operator can retain more plate texture features, the improved effect is obvious. So for those who use color failure of license plate location and license plate again soble operator method of positioning, can improve the recognition rate.

Tilt correction. License plate correction is an important process between license plate location and character segmentation. The license plate image obtained after the license plate positioning inevitably exists some degree of tilt. This tilt will not only bring difficulties to the next character segmentation, will eventually correct rate of license plate recognition caused by direct impact [9]. Firstly extracted the contour of the plate region, and then extracted by Hough transform is close to levels, high contact ratio of parallel double line segment and calculate the parallel double horizontal Angle theta $\theta$, and then to the area between the double shear transformation, the license plate candidate area tilt correction, can be seen from figure 3 , the license plate has been successfully corrected.

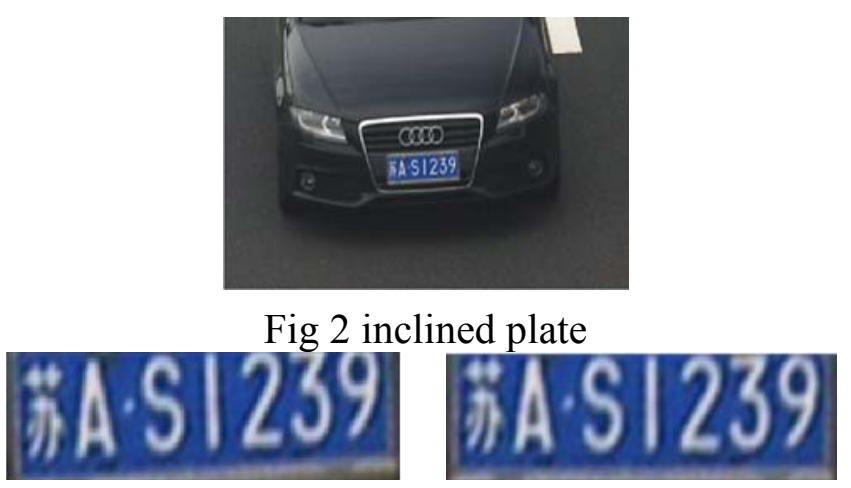

Fig 3 The effect of the correction

\section{Character segmentation}

In the actual process,people are faced with a license character to be recognized,whose background is very complicated. There is a big disturbance and noise, which is caused by uneven lighting and cameras and other equipment, especially skew license.It gives the character segmentation bring some difficulties. The stand or fall of character segmentation will have a direct impact on the credibility of 
the identification. Character segmentation algorithm of this paper is based on the license plate character width and the distance between characters and the characteristics of the rules, can effectively solve the problem of character segmentation inaccurate and license plate askew, binarization processing of license plate.According to prior knowledge, standards of vehicle license (except for military vehicles, police cars, coach cars, consulate car) has seven characters. first abbreviation for province name (Chinese characters), second for English letters, again for English letters or Arabic digits. Total length of the character region is set $\mathrm{m}_{-}$Width, the average estimate of a single character width $\mathrm{m}_{-}$Width / 7.5.Because of the second and third character spacing bigger than any other character spacing, it begin to divide two characters from the left edge of the image license. Then the license right boundary image are segmented consecutively 5 characters.For the factor of uneven illumination and binarization,there is 1 to 2 pixels with the theoretical estimates floating between character width and spacing in the actual binarization license plate image.According to the results of the coarse segmentation and license plate character of law, it introduced a reasonable segmentation template.It is divided into seven pieces,making the vertical projection and segmentation template matching, to achieve the perfect combination. So it can accurately license plate character segmentation,segmentation results as shown 4 shown.

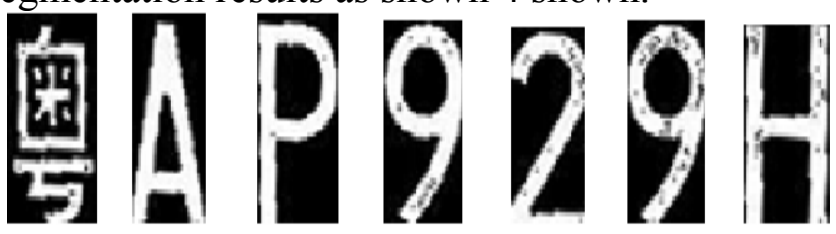

Fig. 4 Character segmentation result

\section{Character recognition}

This article through the analysis of the difficulty of license plate recognition of China as well as the commonly used method of license plate recognition. Then, according to the particularity of Chinese license plate recognition system, license plate character recognition using BP neural network algorithm [10] to achieve character recognition of license plate recognition system, in order to improve the efficiency of license plate recognition, with the method of multi network classifier, which are respectively set up Chinese characters recognition network, letter recognition and alphanumeric recognition network. According to the result of experiment shows that this algorithm is efficient, faster recognition and has good anti-interference, and can satisfy the requirement of high recognition rate.

\section{Experimental results and analysis}

Above describes the detailed process of this algorithm, now, The proposed method is verified by the image obtained in the natural environment, and the results are shown in Fig 5.Fig 5 (a) - Fig 5 (e) is the result of a single recognition of different sizes, different angles of the plate in different natural background; Fig 5 (f) - Fig 5 (g) is the same picture image in the presence of multiple license plates in complex natural background in the recognition results, which Fig 5 (f) is a stationary vehicle image, Fig 5 (g) highway motor vehicle images, extracted from the results can be seen, license plate due to the motion is blurred. Fig (a), Fig 5 (b) cloudy shooting, Fig 5 (d) for the night shooting, Fig 5 (e) for the foggy days, the rest of the images for the sunny. As can be seen from Figure5, this method is able to successfully recognition and segment the license plate region in the presence of multi license plates, different license plates, different location, different illumination and complex environment.

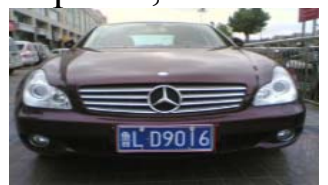

(1) Original image

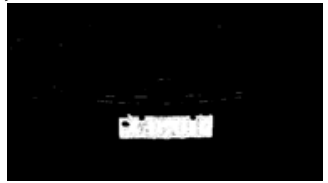

(2) after treatment

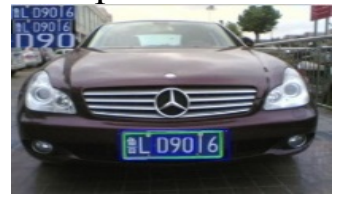

(3) Results

(a) no tilt, large font, a single license plate recognition 


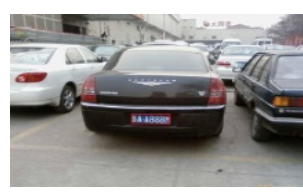

(1) Original image

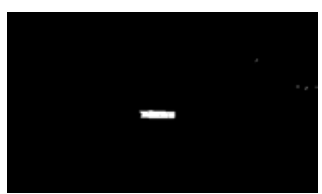

(2) after treatment

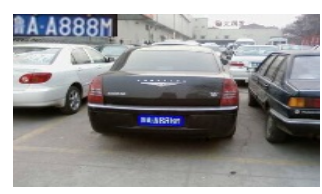

(3) Results

(b) the background is complex, no tilt, small font, a single license plate recognition

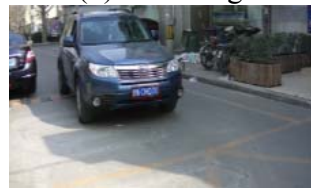

(1) Original image

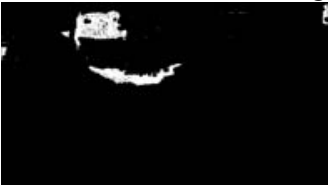

(2) after treatment

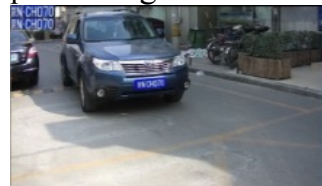

(3) Results

(c) the background is complex, the inclination is small, the license plate and the vehicle body color is

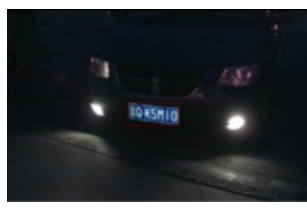

(1) Original image similar, the single license plate recognition

(d) the background is complex, the tilt is small, the recognition of a single license plate at night

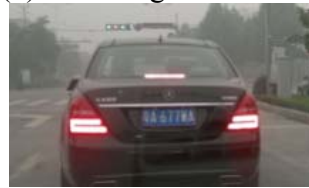

(1) Original image

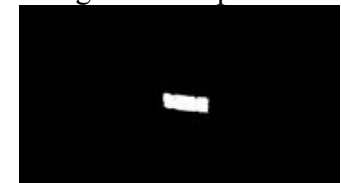

(2) after treatment

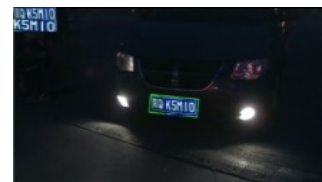

(3) Results

(E) the background is complex, no tilt, the recognition of a single license plate on a cloudy day

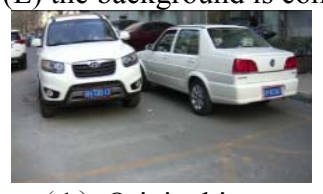

(1) Original image

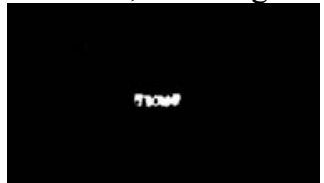

(2) after treatment

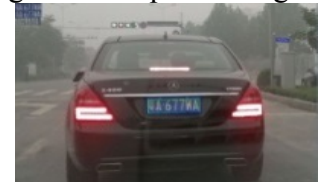

(3) Results

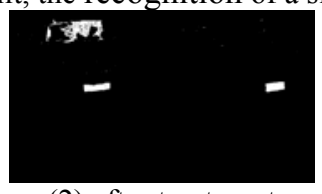

(2) after treatment

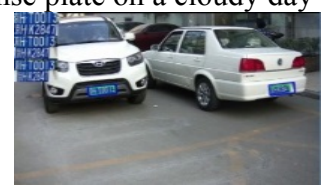

(3) Results

(f) the background is complex, the license plate size is different, the angle of inclination is different, the

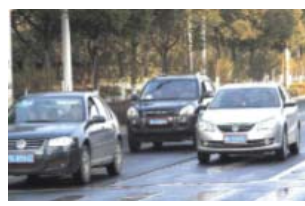

(1) Original image

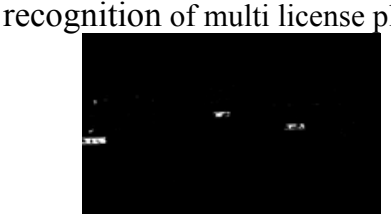

(2) after treatment

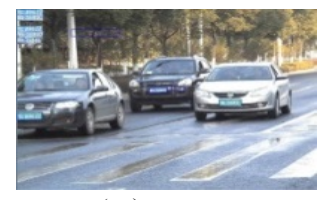

(3) Results

(g) the background is complex, the license plate is small, moving image, multi license plate recognition

Fig. 5 effect of vehicle license plate recognition under complex natural environment

We will be based on HSV color space of license plate recognition method [11], based on the license plate recognition method is edge detection and mathematical morphology method of license plate recognition [12] and is presented in this paper are compared and the test results are shown in Table 2 shows.

Table2 Comparision results of accuracy of three recognition methods

\begin{tabular}{cccc} 
Recognition method & $\begin{array}{c}\text { Comprehensive } \\
\text { method of license } \\
\text { plate recognition } \\
\text { based on HSV } \\
\text { color space }\end{array}$ & $\begin{array}{c}\text { Edge detection and } \\
\text { mathematical } \\
\text { morphology-based } \\
\text { approach }\end{array}$ & $\begin{array}{c}\text { The method } \\
\text { proposed in this } \\
\text { paper }\end{array}$ \\
\hline Bicense plate types license plate $(800)$ & $93.1 \%(745)$ & $87.6 \%(681)$ & $95.7 \%(760)$ \\
Yellow license plate $(600)$ & $94.6 \%(568)$ & $88.2 \%(529)$ & $95.96 \%(576)$ \\
Multi license plate $(500)$ & $90.1 \%(451)$ & $80.5 \%(403)$ & $95.1 \%(476)$ \\
\hline
\end{tabular}




\section{Summary}

With the mentioned in the introduction of other compared to the method of license plate recognition based on color, this method not only solve the using of license plate background to license plate recognition accuracy rate is not high, the basic problem, and proposes a combined with the color features of the plate, the geometric and gray features of license plate recognition method, which can handle more than one image to the plate as well as different types of license plates, but also for the image of the vehicle license plate prior knowledge and background in your environment requires less, it has better adaptability and flexibility. Through the analysis of the experimental results, it can be known that the recognition accuracy of the proposed algorithm is more than $95 \%$ for all kinds of vehicle license plates with complex background. So this algorithm can be applied to the vehicle license plate recognition system, and it can also be applied to other related fields.

\section{References}

[1] Guo Jie,Shi Peng Fei. License plate location method based on color and texture analysis [J]. Chinese journal of image and graphics: A series, 2002, 7 (5) : 472-476.

[2] Dai Qing Yun,Yu Ying Lin. A license plate image segmentation method based on wavelet and morphology [J]. Chinese journal of image and graphics: A series, 2000, 5 (5) : 411-415.

[3] Zuo Qi, Shi Zhong Ke. A real-time license plate image segmentation method based on mathematical morphology [J]. Chinese journal of image and graphics: A series, 2004, 8 (3) : 281-285.

[4] Li Gang, Zeng Yue Li, Lin Ling, etc. The license plate location algorithm based on mathematical morphology [J]. Journal of instruments and meters, 2007, 28 (7) : 1323-1327.

[5] Zhang Yin, Pan Yun He. Color car new image segmentation method [J]. Chinese journal of image and graphics: A series, 2001, 6 (4) : 374-377.

[6] Kamat V, Ganesan s. An efficient implementation of the Hough transform for detecting vehicle license plates using DSP 'S [C] / / Real - Time Technology and Applications Symposium, 1995. Proceedings. IEEE, 1995, 58-59.

[7] Li H, Qin t. A license plate location algorithm -based on multi - component edge, A combination of the HSI color space [C] / / Image and Signal Processing (CISP), 2011, 4 th International meets on. IEEE, 2011, 2:1127-1129.

[8] Zhang Yu Jin. Engineering (I), image processing and image analysis (series teaching materials of electronics and information technology, Tsinghua University) [J]. 1999.

[9] Zhang Jin Yan. The license plate recognition algorithm based on neural network research [D]. Yanshan university, 2010.

[10] Chen Jing Lai. License plate recognition based on neural network method research [D]. Shenyang architecture university, 2012.

[11] Xie Yong Xiang, Dong Lan Fang. License plate recognition method based on HSV space and template matching study [J]. Journal of graphics, 2014, 35 (4) : 585-589.

[12] Zhao Jun Mei, Zhang Li Ping. License plate recognition method based on mathematical morphology [J]. Vehicle and power technology, 2008 (4) : 31-34. 